\title{
Variations in the arteries of upper limb- an ontogenic explaination
}

\author{
Preeti Sonje, Dinesh Patel, Vasanti Arole, P Vatsalaswamy \\ Padmashree Dr D Y Patil Medical college Pune, DY Patil University \\ *Corresponding author E-mail: drpreetisalgar @ gmail.com
}

\begin{abstract}
During routine dissection in the department of Anatomy, Padmashree Dr.D.Y.Patil Medical College,Pimpri, Pune,variation of brachial artery was found. It was labeled as superficial brachial artery as it was arising from third part of axillary artery and was superficial to nerves and vessels of axilla .This superficial brachial artery was dividing into Radial and Ulnar arteries. Also Common trunk for Subscapular and Anterior circumflex scapular arteries was seen arising from axillary artery. Its embryological basis is also discussed along with details of observations.
\end{abstract}

Keywords: Deep brachial artery, median nerve, superficial brachial artery, sub scapular artery, third part of axillary artery.

\section{Introduction}

During routine dissection in the department of Anatomy, Padmashree Dr. D.Y.Patil Medical College, Pimpri, Pune, variation of brachial artery was found in the right arm of adult male cadaver. A large branch was observed, arising from the third part of axillary artery distal to pectoralis minor and $0.5 \mathrm{~cm}$ proximal to the common trunk of origin of sub scapular and circumflex humeral arteries. This artery was named as superficial brachial artery.

Normally Axillary artery continues as Brachial artery after passing between two roots of median nerve at the lower border of teres major muscle. In the arm it gives Profunda brachii artery so also Superior and Inferior ulnar collateral arteries .It terminates in the cubital fossa at the neck of radius by dividing into Radial and Ulnar arteries.

In the present case the Profunda brachii and Superior ulnar collateral arteries were arising from deep brachial artery, the Inferior ulnar collateral artery was absent whereas the terminal branches namely Radial and Ulnar arteries were arising from superficial brachial artery.

\section{Observations}

In an adult old male cadaver superficial brachial artery was found in right upper extremity. This artery was arising from the third part of axillary artery. The origin of the artery was distal to the lower border of pectoralis minor but $0.5 \mathrm{~cm}$ proximal to the origin of common trunk for subscapular and anterior circumflex humeral arteries which are normally the branches from third part of axillary artery (Fig.1).

Superficial Brachial artery was thick and was passing initially medial and from middle of arm anterior to the median nerve .After reaching the cubital fossa it was bifurcating into Radial and Ulnar arteries at the neck of radius (Fig.2). Common interosseous artery was seen arising from Radial artery. Thus rather than being branches of deep brachial artery, Radial and Ulnar arteries in the present case were the branches of superficial brachial artery.

However Subscapular artery had a peculiar course .After separating from the common trunk, artery was seen passing laterally,winding around the Radial nerve, had a course posterior to Radial and Median nerves, Deep brachial artery and Superficial brachial artery before passing anterior to subscapularis muscle. Deep brachial artery was a continuation of axillary artery This deep brachial artery was very thin seen passing between the two roots of median nerve. Deep brachial artery was giving the branches in the upper arm as follows

- Two Profunda Brachii arteries which were very thin.

- Superior Ulnar collateral artery.

- Muscular branches to the Biceps brachii muscle (Fig.3). 


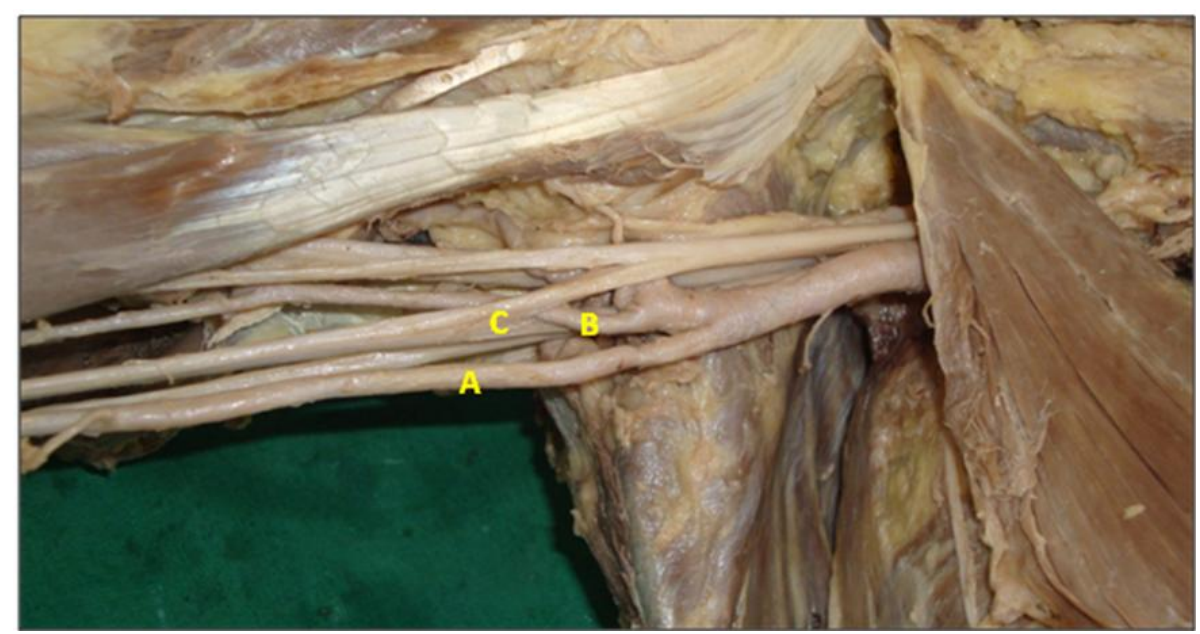

Fig. 1: A-Superficial brachial artery, B - Deep brachial artery passing between two roots of median nerve $(\mathrm{C})$. C - Median nerve.

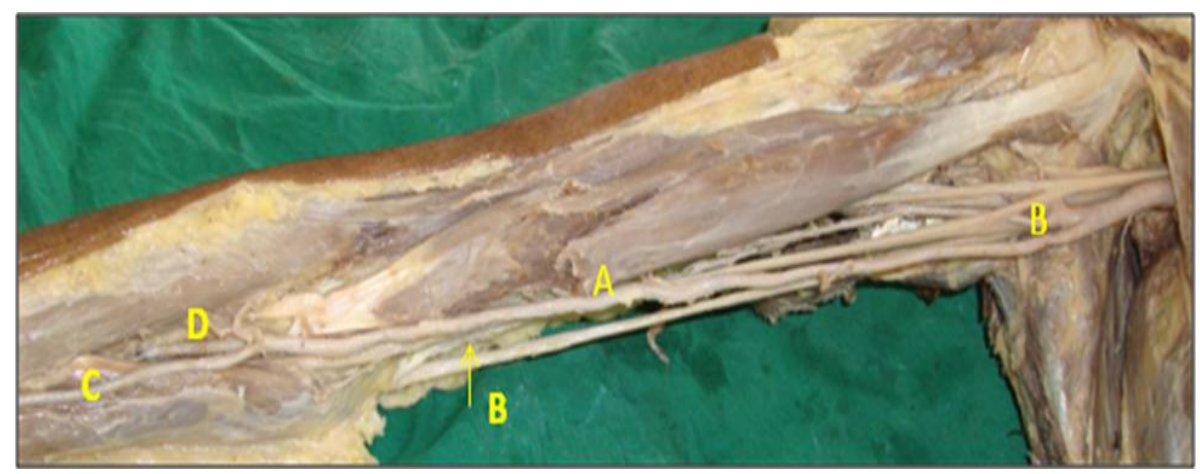

Fig. 2: A- tortuous superficial brachial artery, which was dividing into C -Ulnar and D - Radial arteries at the level of the neck of radius .B- Deep brachial artery was seen ending at the elbow.

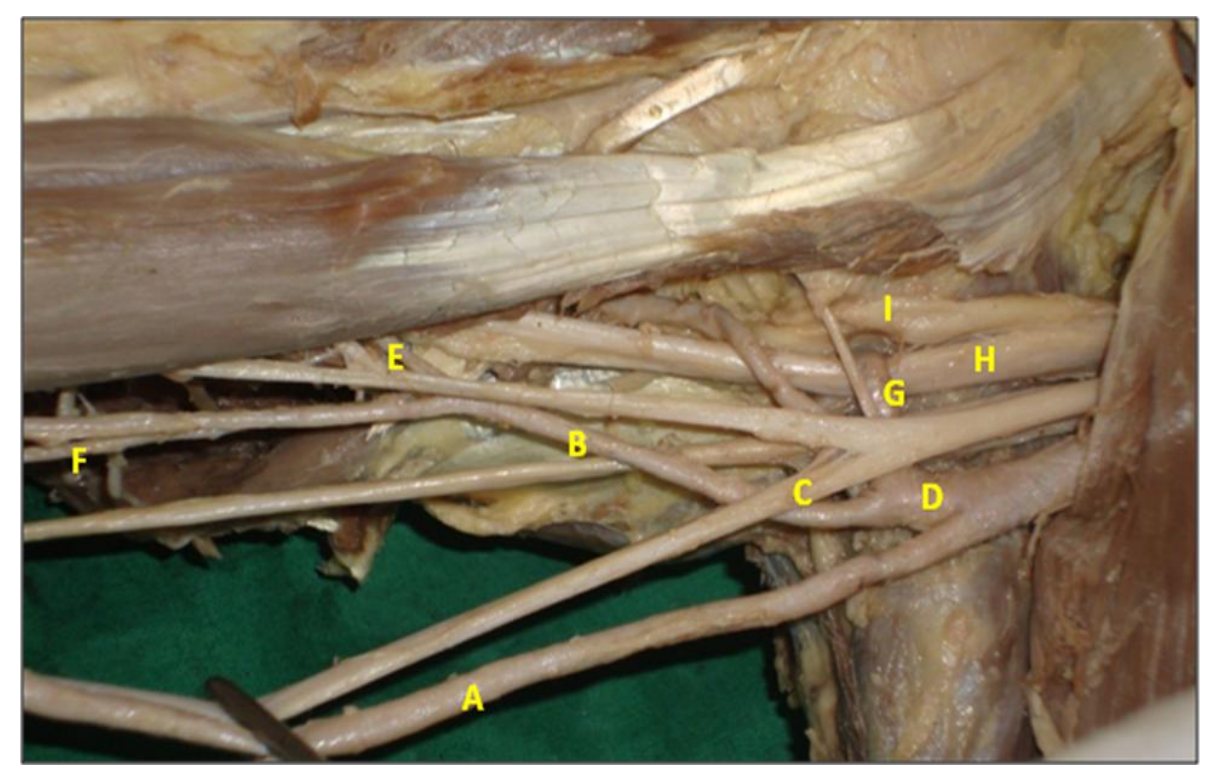

Fig. 3 A- superficial brachial artery ,B- Deep brachial artery ,D -Common trunk for the origin of Anterior circumflex humeral and sub scapular arteries, E - Two Profunda brachii arteries seen as branches of deep brachial artery. F-Superior ulnar collateral artery seen as branch of deep brachial artery.G- Subscapular artery, H- Radial nerve, I- Axillary nerve. 


\section{Discussion}

In the present case superficial brachial artery was tortuous and running medial to the median nerve in the axilla. Deep brachial artery was giving branches as Profunda brachii and Superior ulnar collateral arteries. Superficial brachial artery was dividing into Radial and Ulnar arteries at the neck of radius.Common interosseous artery was seen as a branch of radial artery. Further course of ulnar and radial arteries was normal in the forearm and hand. KawashimaT et al [1] have found the Superficial brachial artery passing above the cords of brachial plexus .

Yalcin B et al [2] have studied superficial brachial artery as an important vessel in fetal life for replacing or supporting the definitive brachial artery.

Yang Hee-Jun et al [3] found the superficial brachial artery (SBA), as a branch of the axillary artery, is one of the most common arterial variations. He has classified the superficial brachial artery according to its origin from the Axillary artery. As per this classification Superficial brachial artery in the present case can be classified as belonging to class I a. Neelamjit Kaur et al [4] also reported bilateral superficial brachial artery terminated by dividing into Radial and Ulnar arteries on left side, while on the right side it divided into Radial and Ulnar arteries and the latter continued as common interrosseous artery in the forearm.

Kanika Sachdeva et al [5] found bilateral superficial brachial arteries crossing superficial to median nerve.

S. Anagnostopoulou et al [6] found the presence of a superficial brachial artery which terminated by dividing into 3 branches at the cubital fossa namely Radial, Ulnar and Common interosseous arteries.

Subscapular artery winding round the Radial nerve has been reported for the first time in the present case.

Formation of Superficial brachial artery can be explained ontogenically as follows.

Ontogenic explanation of development of right upper limb arteries as described by Singer [7] Quoted by Patnaik VVG [8].

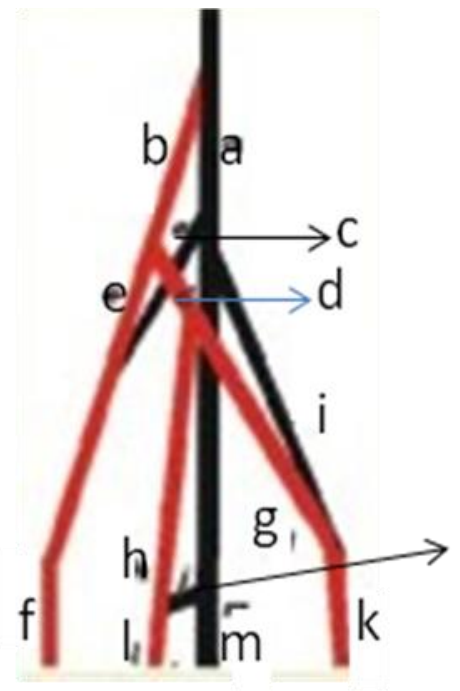

A

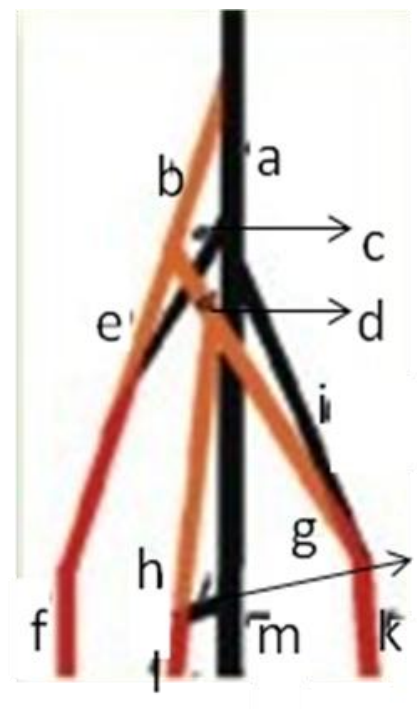

B Fig 4

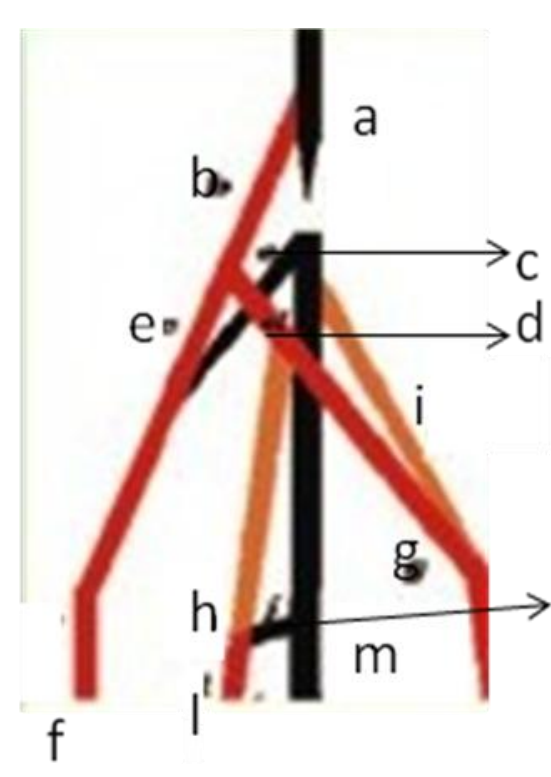

C

\section{A-Embryonic arteries}

a - primitive axial artery

(e,m,i) - Lateral, median and medial branches of axillary artery.

b - Superficial brachial artery.

$(\mathrm{c}, \mathrm{f}),(\mathrm{d}, \mathrm{g}),(\mathrm{h}, \mathrm{i})$ - lateral , medial and median branches of Superficial brachial artery.

\section{B- Normal definitive arteries}

Segments b,c,d,h,g regress

(i+k) - Ulnar artery

(m) - Common interosseous artery

(e+f) - Radial artery 


\section{C-Present case}

Distal segment of ' $a$ ' so also segments $\mathrm{i}$ and h regress.

b- Superficial brachial artery

$(c+f)-$ Radial artery

$(\mathrm{d}+\mathrm{g}+\mathrm{k})-$ Ulnar artery

Thus Radial and Ulnar arteries are seen as branches of superficial brachial artery.

$(\mathrm{e}+\mathrm{m})$-Common interosseous artery, seen as a branch of Radial artery instead of branch of Ulnar artery as is normally seen.

\section{Conclusion}

deep brachial artery was seen to be giving branches as Profunda brachii and Superior ulnar collateral arteries and ending at the elbow. Superficial brachial artery was seen to be tortuous passing medial and then crossing superficial to Median nerve and dividing into Radial and Ulnar arteries at the neck of the radius. Common interosseous artery was arising from Radial artery instead of Ulnar artery. Knowledge of Superficial brachial artery is important to avoid injury to the artery during any surgical or diagnostic procedures. Also there was a common trunk for Sub scapular and anterior circumflex humeral arteries.Subscapular artery having a peculiar course passing laterally and winding around the radial nerve, which may cause compression effects of the radial nerve.

\section{References}

[1] Kawashima T, Yoshitomi S, Sasaki H.Anatomical relationship between the superficial brachial arteries and the brachial plexus in humans, and their morphological significance. Folia morphol,63(2004):465-471(Pubmed)

[2] Yalcin B,Yazar F(2006).Arterial variations of the upper extremities.Anat sci.int.;81:62-64.

[3] Yang Hee-Jun ,H J Yang, YC Gil, WS Jung, HY Lee.Variations of the Superficial Brachial Artery in Korean Cadavers .Journal of Korean Medical Science ;23(5) (2008):884-887.

[4] Kaur Neelamjit et al.. Bilateral superficial brachial artery - a case report.International journal of anatomical variations4(2011): 207-210

[5] Sachdeva Kanika ,Sharma T ,Singla R K . Bilateral superfi cial brachial artery .Kathmandu University Journal Vol. 7, No. 4, Issue 28, (2009); 426-428

[6] S. ANAGNOSTOPOULOU, D. VENIERATOS.An unusual branching pattern of the superficial brachial artery accompanied by an ulnar nerve with two roots. Journal of Anatomy,Volume 195, Issue 3. (October 1999), pages 471-476.

[7] Singer E. Embryological pattern persisting in the arteries of the arm. Anatomical Record. 1933; 55: 403-409 Quoted by Patnaik. (Vide infra).

[8] Patnaik VVG, Kalsey G, Singla RK. Branching pattern of brachial artery - a morphological study. J Anat Society of India.2002, 51: 176-18

[9] Rodriguez-Baeza A, Nebot J, Ferreira B, Reina F, Perez J, Sanudo JR, Roig M .Ananatomical study and ontogenetic explanation of 23 cases with variations in the main pattern of the human brachio-antebrachial arteries. J Anat.; 187(1995): 473-479. 